\title{
Privatabrechnung
}

\section{Bahn frei für die neue GOÄ!}

\section{Der Deutsche Ärztetag hat be- schlossen, die Gespräche mit}

PKV und Politik über die GOÄ-Reform neu zu starten. Der neue Ausschussvorsitzende Dr. Klaus Reinhardt erklärt, wie es nun weitergeht.

_ Die ärztlichen Verbände haben auf dem Ärztetag ihre Differenzen im Detail zugunsten gemeinsamer, klar formulierter Forderungen überwunden - ein Zeichen der Geschlossenheit. Dies war auch möglich, weil die Führung der Bundesärztekammer zuvor die Reißleine gezogen, den Reset-Knopf gedrückt und den Weg für neue Verhandlungen mit dem PKV-Verband und dem Gesundheitsministerium frei gemacht hatte.

Als neuer Vorsitzender des GOÄ-Ausschusses der Bundesärztekammer habe ich deutlich gemacht, dass wir von nun an den Entwurf einer Gebührenordnung gemeinsam mit den Fach- und Berufsverbänden erstellen werden, bevor wir unseren Vorschlag formal in die Verhandlungen einbringen. Dabei werden wir uns alle notwendige Zeit lassen und am Ende im ärztlichen Interesse einen vernünftigen Kompromiss finden zwischen angemessenen Forderungen und Durchsetzbarem.

\section{Persönliche ärztliche Zuwendung wird gestärkt}

Die neue GOÄ ist nicht nur eine Chance, überholte Strukturen aufzubrechen und Leistungen auf dem neuesten medizinischen Stand abzubilden, statt weiter mit Analogziffern arbeiten zu müssen. Sie ist vor allem auch die Gelegenheit, mit Blick auf lange unterbewertete Leistungen klare Signale zu setzen. Dies gilt insbesondere für den Bereich der persönli- chen ärztlichen Zuwendung. Die erwartbaren Zuwachsraten werden zunächst moderat ausfallen, der Vorteil der Rechtssicherheit bei der Abrechnung moderner Leistungen wird dies aber deutlich kompensieren.

Ein Wort vielleicht noch zur gemeinsamen Kommission mit den Privatkassen, die im Vorfeld für viel Aufregung gesorgt hat. Ich persönlich glaube vor allem an die Chancen der Kommission, neue Leistungen zeitnah einbringen und angemessen bewerten zu können. Ansonsten gilt für mich der Ärztetagsbeschluss: Änderungen sowohl des Paragrafenteils der GOÄ als auch der Bundesärzteordnung sollte es nur geben, wenn sie inhaltlich dem Ziel einer novellierten Gebührenordnung dienen. Dieser Kompromiss lässt uns die notwendigen Spielräume.

Der Deutsche Ärztetag war so gesehen nicht nur ein erfolgreicher Schritt hin zu einer neuen GOÄ, er war auch ein wichtiger Beweis dafür, dass die deutsche Ärzteschaft die Gesundheitspolitik konstruktiv und entschlossen mitgestalten kann. Ich wünsche mir sehr, dass insofern auch ein Signal in Richtung KVSystem ausgeht. Das Damoklesschwert weiteren staatlichen Eingreifens in unsere Selbstverwaltung muss wieder verschwinden. Ansonsten wäre das Fundament unserer Freiberuflichkeit auf Dauer ernsthaft gefährdet.

Dr. Klaus Reinhardt
Springer 